Editor's Note: These short, critical reviews of recent papers in the Journal, written exclusively by graduate students or postdoctoral fellows, are intended to summarize the important findings of the paper and provide additional insight and commentary. For more information on the format and purpose of the Journal Club, please see http://www.jneurosci.org/misc/ifa_features.shtml.

\title{
Bidirectional Changes in the Intrinsic Excitability of Infralimbic Neurons Reflect a Possible Regulatory Role in the Acquisition and Extinction of Pavlovian Conditioned
} Fear

\author{
Christine A. Rabinak, ${ }^{1 *}$ Joshua M. Zimmerman, ${ }^{\star *}$ Chun-hui Chang, ${ }^{1 \star}$ and Caitlin A. Orsini ${ }^{1 *}$ \\ ${ }^{1}$ Department of Psychology and ${ }^{2}$ Neuroscience Graduate Program, University of Michigan, Ann Arbor, Michigan 48109-1043 \\ Review of Santini et al. (http://www.jneurosci.org/cgi/content/full/28/15/4028)
}

\begin{abstract}
Although being able to recall highly feararousing events can be adaptive in certain contexts, it is also advantageous to be able to inhibit these responses. Pavlovian fear extinction occurs when a conditioned stimulus is repeatedly presented in the absence of the unconditioned stimulus, which reduces conditioned fear responses to the stimulus (Maren, 2001). Failure in extinction can lead to the development of psychological disorders such as posttraumatic stress disorder. Therefore, many researchers have focused on the neural processes behind extinction of maladaptive fears.
\end{abstract}

The medial prefrontal cortex is a critical component of the neural circuitry mediating fear acquisition and extinction. Specifically, the infralimbic cortex (IL) is important for consolidation and retrieval of extinction memories and consequent inhibition of conditioned responses (Quirk et al., 2006; Quirk and Mueller,

\footnotetext{
Received June 11, 2008; revised June 3, 2008; accepted June 3, 2008.

We thank our advisor, Dr. Stephen Maren, for comments during preparation of this manuscript and for his invaluable mentorship. Additionally, we thank the University of Michigan's Biopsychology and Neuroscience Journal Club for their comments and suggestions.

${ }^{*}$ C.A.R., J.M.Z., C.-h.C., and C.A.O. contributed equally to this work.

Correspondence should be addressed to Christine Rabinak, Department of Psychology, University of Michigan, 530 Church Street, Ann Arbor, MI 48109-1043. E-mail: rabinak@umich.edu.

DOI:10.1523/JNEUROSCI.2130-08.2008

Copyright $\odot$ 2008 Society for Neuroscience $\quad$ 0270-6474/08/287245-03\$15.00/0
}

2008) (but see Garcia et al., 2006). It has been proposed that extinguished conditioned stimuli activate IL neurons that project to inhibitory intercalated cells in the amygdala. Intercalated neurons consequently inhibit output neurons in the central nucleus of the amygdala (CEA), a structure that mediates the expression of fear. Ultimately, activity in this prefrontal-amygdala pathway is thought to reduce the expression of conditioned fear responses after extinction (Quirk et al., 2003; Paré et al., 2004) (Fig. 1A). The expression of extinction is also context dependent, and hippocampal modulation of either the prefrontal cortex or amygdala may mediate this phenomenon (Corcoran and Maren, 2001; Maren and Quirk, 2004).

On a cellular level, it has recently been demonstrated that neurons in the prelimbic area (PL) of the medial prefrontal cortex display burst firing that is correlated with fear acquisition and expression, whereas neurons in the IL display bursting that is correlated with extinction. Indeed, high-frequency bursting in the IL predicts the degree of extinction retrieval (BurgosRobles et al., 2007; Quirk and Mueller, 2008). Furthermore, NMDA receptors mediate IL bursting, which is hypothesized to induce calcium currents in the IL for consolidation of extinction memory (Quirk et al., 2006). This suggests that po- tentiation of IL neurons is necessary for both enhancing consolidation of extinction memory and suppressing conditioned fear responses during retrieval of those memories. However, the specific cellular mechanisms that mediate this effect remain unclear. To address this issue, Santini et al. (2008) used intracellular recordings in brain slices to investigate whether extinction of fear is associated with changes in the excitability of IL neurons. They hypothesized that extinction training would increase the intrinsic excitability of IL neurons and consequently lead to an enhancement of spike firing in the IL.

Their experimental design consisted of four groups of rats [Santini et al. (2008), their Fig. $1 A$ (http://www.jneurosci.org/ cgi/content/full/28/15/4028/F1)]. On day 1 , conditioned (COND) and extinguished (EXT) groups received pavlovian fear conditioning consisting of three tonefootshock pairings. On day 2, rats in the EXT group received 10 tone-alone presentations in the conditioning context to extinguish fear acquired the previous day, whereas the COND group remained in their home cages. Additionally, two control groups were included: a NAIVE group that received neither training nor extinction, and a pseudoconditioned (PSEUDO-COND) group that received unpaired tone-shock presentations dur- 
ing training and no extinction session. On day 3 , rats in all groups received two tonealone presentations during a retention test; rats in the COND and EXT groups received these tones in the conditioning chamber, whereas rats in the PSEUDOCOND and NAIVE groups received the tones in a novel context. As expected, during testing, COND animals froze significantly more than the other groups, whereas EXT animals froze less than the COND groups, but more than both NAIVE and PSEUDO-COND groups [Santini et al. (2008), their Fig. $1 B$ (http:// www.jneurosci.org/cgi/content/full/28/ 15/4028/F1)].

Immediately after the final test, the animals were killed and cortical brain slices were taken for current-clamp recordings in the IL and the PL. In the IL, neurons in the COND group fired significantly less in response to current injection than did IL neurons recorded from rats in NAIVE, PSEUDO-COND, and EXT groups [Santini et al. (2008), their Figs. 2C,D (http:// www.jneurosci.org/cgi/content/full/ 28/15/4028/F2), $5 A, B$ (http://www. jneurosci. org/cgi/content/full/28/15/ 4028/F5)]. Indeed, extinction training appeared to reverse the conditioning-related decrease in firing, bringing firing in the EXT group to levels indistinguishable from those in the NAIVE and PSEUDOCOND groups [Santini et al. (2008), their Figs. $1 B$ (http://www.jneurosci.org/ cgi/content/full/28/15/4028/F1), 2C,D (http://www.jneurosci.org/cgi/content/ full/28/15/4028/F2), 5A,B (http://www. jneurosci.org/cgi/content/full/28/15/4028/ F5)]. It should be noted that the authors' design does not eliminate the possibility that mere tone exposure (rather than extinction per se) contributes to these changes.

Although IL spiking did not differ between NAIVE, PSEUDO-COND, and EXT groups, the firing pattern in IL neurons from EXT rats showed significantly more bursting activity [Santini et al. (2008), their Fig. 3 (http://www.jneurosci. org/cgi/content/full/28/15/4028/F3)]. These variations in spiking were attributed to decreases in the fast afterhyperpolarization [Santini et al. (2008), their Fig. 4 (http://www.jneurosci.org/cgi/content/ full/28/15/4028/F4)], which suggests that changes in the excitability in IL neurons may be attributable to activation of calcium-activated potassium channels (e.g., SK channels). Calcium influx through NMDA receptors may be an essential step in the regulation of the fast afterhyperpolarization by SK channels (Quirk et al., 2006). Interestingly, fear conditioning

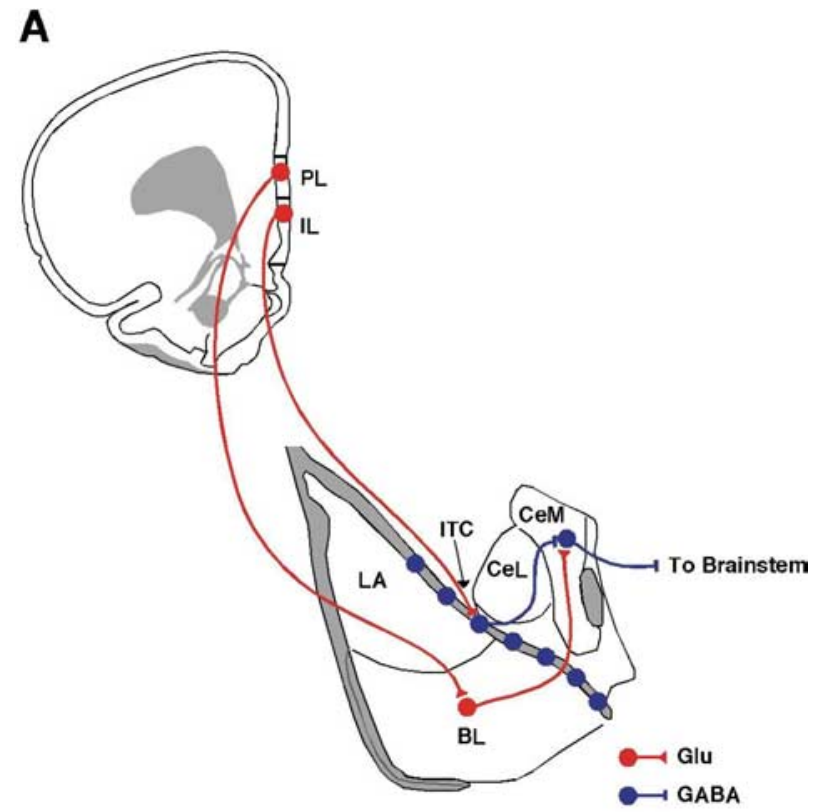

B Before Extinction

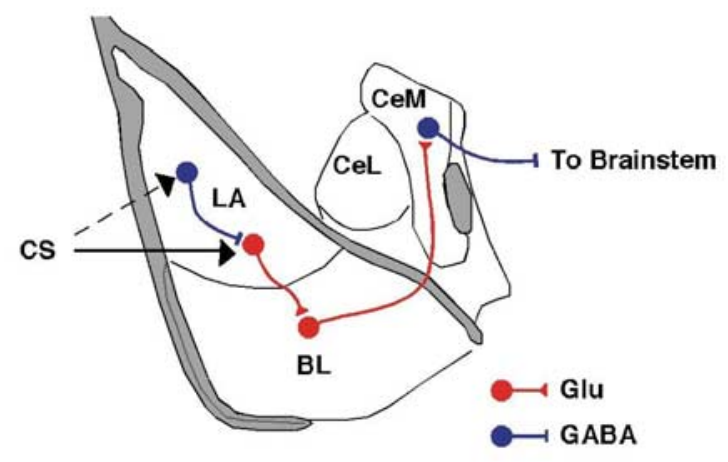

After Extinction

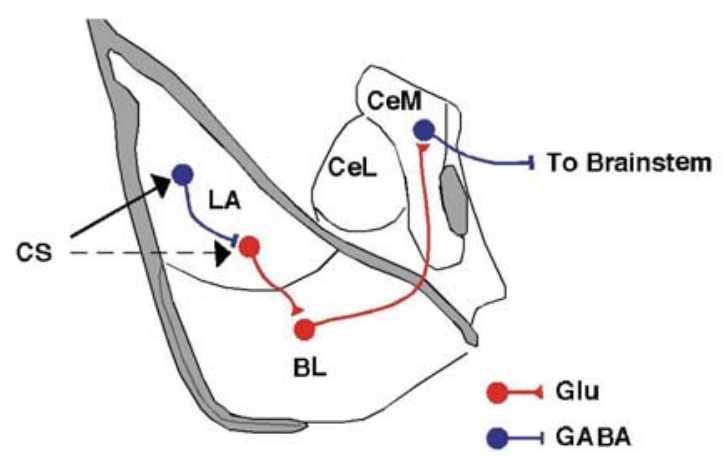

Figure 1. A, Schematic of the medial prefrontal cortex projections to the amygdala hypothesized to underlie extinction. The IL excites GABAergic intercalated neurons, which in turn inhibit GABAergic cells in the centromedial amygdala (CeM). The PL excites glutamatergic neurons in the basolateral amygdala (BL), which excite GABAergic neurons in the CeM. $\boldsymbol{B}$, Schematic of the relative contribution of the conditioned stimulus input into the amygdala before and after extinction. Before extinction, input about the conditioned stimulus to the amygdala generates increased firing in projection neurons, which promotes freezing behavior (top). After extinction, the conditioned stimulus strongly activates interneurons (bottom), thereby inhibiting freezing behavior. GABAergic pathways are in blue, and glutamatergic pathways are in red. ITC, Intercalated nuclei; CeL, centrolateral amygdala. This figure was adapted from Swanson (1992). 
was associated with a decrease in the slow afterhyperpolarization, an effect that accounts for decreases in IL excitability in conditioned rats.

Bursting in IL neurons may be important for fear suppression after extinction insofar as low levels of freezing were associated with high levels of bursting [Santini et al. (2008), their Fig. 3D (http://www. jneurosci.org/cgi/content/full/28/15/ $4028 / F 3)]$. It is possible that burst firing in the IL is particularly effective at exciting inhibitory intercalated cells in the amygdala, which then inhibit fear expression via the CEA. Likewise, fear conditioning may decrease IL excitability to remove tonic inhibition of the amygdala and facilitate the expression of conditioned fear. This suggests that the IL is positioned to exert bidirectional control over fear expression, enhancing it after conditioning and suppressing it after extinction.

Interestingly, Santini et al. (2008) did not find changes in the excitability of PL neurons after either conditioning or extinction [Santini et al. (2008), their Fig. 6 (http://www.jneurosci.org/cgi/content/ full/28/15/4028/F6)]. This outcome does not appear to be congruent with recent reports that inactivation of the PL impairs the expression of fear (Corcoran and Quirk, 2007) and that electrical stimulation of the PL enhances the expression of fear (Vidal-Gonzalez et al., 2006). These inconsistencies could be attributable to different ways of assessing the role of PL in fear expression (electrophysiology vs pharmacological manipulations) or different testing models (delayed vs trace conditioning). Alternatively, fear conditioning may alter synaptic transmission in the PL, as opposed to enhancing the intrinsic cellular excitability of PL neurons.

According to the circuitry described above (Fig. 1A), the IL regulates the expression of fear-conditioned responses by inhibiting the CEA, the major descending output of the amygdala. An alternative is that the modulation of fear responses may occur upstream of the CEA in the lateral nucleus of the amygdala (LA), which is the primary sensory interface of the amygdala. Indeed, neurons in the LA fire less in response to the conditioned stimulus during the course of extinction (Quirk and Mueller, 2008). Moreover, Maren and Hobin (2007) have shown that decreases in LA spike firing after extinction are context dependent, and that both LA firing and conditional fear can be renewed when animals are exposed to a conditioned stimulus outside the extinction context. It is therefore possible that extinction is associated with plasticity among inhibitory interneurons in the LA, and that the balance of excitation and inhibition in the LA ultimately regulates the transmission of conditioned stimulus information to the CEA (Fig. $1 B$ ). It is not unreasonable to think that the prefrontal cortex could regulate this process as well. Indeed, modulation of neuronal activity in both the CEA and LA may contribute to the regulation of fear after extinction, and the relative contribution of each area to the expression of extinction requires further investigation.

Although the role of the medial prefrontal cortex in the expression of conditioned fear has been examined previously, this is the first study that combined classical fear conditioning and patch-clamp electrophysiology to investigate the plasticity underlying fear extinction. In accordance with several previous studies (Quirk and Mueller, 2008), Santini et al. (2008) provide further cellular evidence that the IL regulates fear expression through bidirectional changes in intrinsic excitability after conditioning and extinction. Importantly, they present a compelling argument for the role of plasticity specifically within the IL that likely contributes to the memory formed during extinction. Better understanding the cellular mechanisms of fear regulation is critical for developing optimal clinical interventions for psychological trauma in humans.

\section{References}

Burgos-Robles A, Vidal-Gonzalez I, Santini E, Quirk GJ (2007) Consolidation of fear extinction requires NMDA receptor-dependent bursting in the ventromedial prefrontal cortex. Neuron 53:871-880.

Corcoran KA, Maren S (2001) Hippocampal inactivation disrupts contextual retrieval of fear memory after extinction. J Neurosci 21:1720-1726.

Corcoran KA, Quirk GJ (2007) Activity in prelimbic cortex is necessary for the expression of learned, but not innate, fears. J Neurosci 27:840-844.

Garcia R, Chang CH, Maren S (2006) Electrolytic lesions of the medial prefrontal cortex do not interfere with long-term memory of extinction of conditioned fear. Learn Mem 13:14-17.

Maren S (2001) Neurobiology of pavlovian fear conditioning. Annu Rev Neurosci 24:897-931.

Maren S, Hobin JA (2007) Hippocampal regulation of context-dependent neuronal activity in the lateral amygdala. Learn Mem 14:318-324.

Maren S, Quirk GJ (2004) Neuronal signalling of fear memory. Nat Rev Neurosci 5:844-852.

Paré D, Quirk GJ, Ledoux JE (2004) New vistas on amygdala networks in conditioned fear. J Neurophysiol 92:1-9.

Quirk GJ, Mueller D (2008) Neural mechanisms of extinction learning and retrieval. Neuropsychopharmacology 33:56-72.

Quirk GJ, Likhtik E, Pelletier JG, Paré D (2003) Stimulation of medial prefrontal cortex decreases the responsiveness of central amygdala output neurons. J Neurosci 23:8800-8807.

Quirk GJ, Garcia R, González-Lima F (2006) Prefrontal mechanisms in extinction of conditioned fear. Biol Psychiatry 60:337-343.

Santini E, Quirk GJ, Porter JT (2008) Fear conditioning and extinction differentially modify the intrinsic excitability of infralimbic neurons. J Neurosci 28:4028-4036.

Swanson LW (1992) Brain maps: structure of the rat brain. New York: Elsevier.

Vidal-Gonzalez I, Vidal-Gonzalez B, Rauch SL, Quirk GJ (2006) Microstimulation reveals opposing influences of prelimbic and infralimbic cortex on the expression of conditioned fear. Learn Mem 13:728-733. 